\title{
GÉNEROS DE EULOPHIDAE (HYMENOPTERA: CHALCIDOIDEA) PRESENTES EN EL CULTIVO DE NARANJO EN EL ESTADO DE MORELOS, MÉXICO
}

\author{
GENERA OF EULOPHIDAE (HYMENOPTERA: CHALCIDOIDEA) PRESENT \\ IN ORANGE ORCHARD IN THE STATE OF MORELOS, MEXICO
}

\section{Lucía Teresa FUENTES GUARDiOLA, Oswaldo GARCÍA MARTÍNEZ,* SERgio RenÉ SÁNCHEZ PEÑA Y JORGE CORRALES REYNAGA}

\author{
Universidad Autónoma Agraria Antonio Narro, Departamento de Parasitología Agrícola Calzada Antonio Narro \\ 1923, Buenavista, 25315 Saltillo, Coahuila <lucia_06_18@hotmail.com>. \\ *Autor de correspondencia: <drogarcia@yahoo.com.mx>. \\ Recibido: 07/12/2016; aceptado: 19/05/2017 \\ Editor responsable: Jesús Romero Nápoles.
}

Fuentes G. L.T, García M. O., Sánchez P. S.R. y Corrales R. J. (2017). Géneros de Eulophidae (Hymenoptera: Chalcidoidea) presentes en el cultivo de naranjo en el estado de Morelos, México. Acta Zoológica Mexicana (n.s.), 33(2), 350-354.

RESUMEN. Debido a la importancia económica de los cítricos en el mundo y al uso de especies de Eulophidae en programas de control biológico, se realizaron recolectas quincenales de material entomológico durante 12 meses en una huerta de cítricos en el estado de Morelos, México. Se determinaron 23 géneros de Eulophidae, pertenecientes a las cuatro subfamilias que conforman ésta, diez en Tetrastichinae, nueve en Eulophinae, tres en Entedoninae y uno en Entiinae.

Palabras clave: Eulophidae, control biológico, naranjo, Morelos, México.

\section{INTRODUCCIÓN}

La citricultura representa una actividad de gran importancia económica y social a nivel mundial, ya que los cítricos son cultivados en las regiones de clima tropical y subtropical del mundo, generando una importante derrama económica (Fronfría, 2003). En México la citricultura se practica como actividad económica en 28 entidades federativas, centrándose principalmente en los cultivos de limón, naranja, toronja y mandarina, entre otros, con más de medio millón de hectáreas sembradas (SIAP, 2016), por lo que es importante conocer la entomofauna benéfica presente en estos agroecosistemas. Dentro de los estados citrícolas del país se encuentra Morelos, con una industria citrícola relativamente reciente y en aumento, en los últimos tres años la superficie sembrada de naranja y limón se ha incrementado en un 6 y $19 \%$, respectivamente (SIAP, 2016); además la producción cuenta con calidad
Fuentes G. L.T, García M. O., Sánchez P. S.R., \& Corrales R. J. (2017). Genera of Eulophidae (Hymenoptera: Chalcidoidea) present in orange orchard in the state of Morelos, Mexico. Acta Zoológica Mexicana (n.s.), 33(2), 350-354.

ABSTRACT. Due to the economic importance of citrus in the world and the use of Eulophidae species in biological control programs, biweekly collects of entomological material were performed for 12 months in a citrus orchard of the state of Morelos, Mexico. A total of 23 genera of Eulophidae were determined in the four subfamilies that integrate this family, ten of Tetrastichinae, nine of Eulophinae, three of Entedoninae and one of Entiinae.

Key words: Eulophidae, biological control, orange orchard, Morelos, Mexico.

para incursionar en los mercados internacionales (SosaArmenta et al., 2012).

La presencia de especies de la familia Eulophidae en los ecosistemas naturales y agroecosistemas es esencial porque contribuyen a la diversidad y estabilidad de los mismos (Yefremova, 2007) ya que desempeñan un papel regulador, principalmente de especies fitófagas.

Eulophidae es una de las familias más grandes y diversas de Chalcidoidea, además económicamente muy importante, ya que muchas especies se utilizan en programas de control biológico (Burks et al., 2011). Los eulófidos abundan en las regiones tropicales y templadas, la mayoría se comportan como parasitoides; sin embargo, también incluyen especies con hábitos fitófagos y depredadores (Gauthier et al., 2000). Es una familia con cerca de 4500 especies (Noyes, 2003) que biológicamente presenta un alto grado de variabilidad, ya que tan sólo la subfamilia Tetrastichinae tiene como hospederos a insectos de más 
de 100 familias, además de nematodos y otros organismos (LaSalle, 1994). Las formas parasíticas pueden atacar todos los estadios de desarrollo de los insectos y comportarse como endoparasitoides y ectoparasitoides primarios e incluso como hiperparasitoides (LaSalle, 1994; Gauthier et al., 2000).

Por todo lo anterior, el objetivo de esta investigación fue conocer los géneros de Eulophidae presentes en el cultivo de naranja de la región citrícola del estado de Morelos, específicamente en la localidad de Zacapalco, municipio de Tepalcingo.

\section{MATERIALES Y MÉTODOS}

El material se obtuvo de los muestreos realizados en el área de estudio, en una huerta de 75 hectáreas de naranja Valencia del rancho "El Pochotillo" que se encuentra en la localidad de Zacapalco, Tepalcingo en el estado de Morelos, localizada a una altura de entre 1100 y 1900 m, entre los paralelos $18^{\circ} 27^{\prime}$ y $18^{\circ} 41^{\prime}$ de latitud norte y los meridianos $98^{\circ} 46^{\prime}$ y $99^{\circ} 01^{\prime}$ de longitud oeste; el clima de la zona es cálido subhúmedo con lluvias en verano, el rango de precipitación es de 800 a 1000 mm (INEGI, 2009).

Las recolectas se realizaron durante el periodo comprendido entre julio de 2014 a julio de 2015 con intervalos de 15 días, con un total de 24 muestreos. El método de recolecta utilizado en cada muestreo fue el conocido como "Pyrethrum spraying" descrito por Noyes (1982), seleccionando al azar cuatro árboles por muestreo a los cuales se les colocaba una manta en el suelo de $5 \times 5 \mathrm{~m}$ (100 m² por muestreo) para luego asperjar la copa del árbol con una mezcla de $84 \mathrm{ml}$ de un insecticida piretroide (Cipermetrina) en $25 \mathrm{~L}$ de agua; transcurridos 30 minutos se recolectaban con un pincel los insectos derribados sobre la manta; posteriormente se colocaban en frascos de $100 \mathrm{ml}$ debidamente etiquetados que contenían alcohol etílico al 70\% como conservador. El material se trasladó, para su revisión y determinación, al Laboratorio de Taxonomía de Insectos y Ácaros del Departamento de Parasitología Agrícola de la Universidad Autónoma Agraria Antonio Narro, Campus Saltillo.

En el laboratorio, los adultos de Eulophidae se separaron del resto de los insectos recolectados, para posteriormente montarlos, tanto en porta y cubre objetos o bien sobre triángulos colocados en un alfiler entomológico. La identificación taxonómica se realizó siguiendo las claves a género de LaSalle (1994), Schauff et al., (1997), Hansson (2002), Burks (2003) y Hansson (2009).

\section{RESULTADOS}

Se recolectaron 362 especímenes adultos de 23 géneros, pertenecientes a las cuatro subfamilias de Eulophidae (Cuadro 1).

De Tetrastichinae se obtuvieron diez géneros, nueve de Eulophinae, tres de Entedoninae y uno de Entiinae. Horismenus (Entedoninae) fue el género del que se recolectaron más individuos con un $65.47 \%$, le siguió $G a$ leopsomyia (Tetrastichinae) con 6.08\%, Deutereulophus (Eulophinae) con 5.52\% y Entiinae estuvo representado únicamente por Euderus (0.28\%) (Cuadro 1).

La mayor cantidad de especímenes de Eulophinae se recolectaron en octubre de 2014 y mayo de 2015, sobresaliendo el género Deutereulophus (5.52\%) en estos dos meses. Euplectrus $(2.76 \%)$ se presentó en poblaciones más altas en noviembre (2014) y enero (2015) y Elas-

Cuadro 1. Adultos de Eulophidae recolectados en un huerto de naranja Valencia en Zacapalco, Tepalcingo, Morelos.

\begin{tabular}{|c|c|c|}
\hline Subfamilia & Género & $\begin{array}{c}\text { Núm. de } \\
\text { individuos y \% }\end{array}$ \\
\hline \multirow[t]{9}{*}{ Eulophinae } & Cirrospilus Westwood, 1832 & $2(0.55 \%)$ \\
\hline & Deutereulophus Schulz, 1906 & $20(5.52 \%)$ \\
\hline & Elachertus Spinola, 1811 & $2(0.55 \%)$ \\
\hline & Elasmus Westwood, 1833 & $8(2.21 \%)$ \\
\hline & Euplectrus Westwood, 1832 & $10(2.76 \%)$ \\
\hline & Miotropis Thomson, 1878 & $2(0.55 \%)$ \\
\hline & Paraolinx Ashmead, 1894 & $2(0.55 \%)$ \\
\hline & Pnigalio Schrank, 1802 & $2(0.55 \%)$ \\
\hline & Sympiesis Förster, 1856 & $1(0.28 \%)$ \\
\hline \multirow[t]{3}{*}{ Entedoninae } & Chysocharis Förster, 1856 & $3(0.83 \%)$ \\
\hline & Horismenus Walker, 1843 & $237(65.47 \%)$ \\
\hline & Pediobius Walker, 1846 & $9(2.49 \%)$ \\
\hline Entiinae & Euderus Haliday, 1844 & $1(0.28 \%)$ \\
\hline \multirow[t]{10}{*}{ Tetrastichinae } & Aprostocetus Westwood, 1833 & $11(3.04 \%)$ \\
\hline & Baryscapus Förster, 1856 & $11(3.04 \%)$ \\
\hline & Eriastichus LaSalle, 1994 & $1(0.28 \%)$ \\
\hline & Galeopsomyia Girault, 1916 & $22(6.08 \%)$ \\
\hline & Paragaleopsomyia Girault, 1917 & $1(0.28 \%)$ \\
\hline & Pentastichus Ashmead, 1894 & $1(0.28 \%)$ \\
\hline & Quadrastichus Girault, 1913 & $3(0.83 \%)$ \\
\hline & Sigmophora Rondani, 1867 & $2(0.55 \%)$ \\
\hline & Tamarixia Mercet, 1924 & $10(2.76 \%)$ \\
\hline & Tetrastichus Haliday, 1844 & $1(0.28 \%)$ \\
\hline 4 & 23 & $362(100 \%)$ \\
\hline
\end{tabular}


mus (2.21\%) se encontró con mayor frecuencia en junio (2015), estos tres géneros fueron los más recolectados de esta subfamilia (Fig. 1).

La distribución estacional mostró que fue muy notoria la presencia de Horismenus (65.47\%) en todos los meses muestreados, excepto agosto y que el número más alto de especímenes de este género se obtuvo en octubre; los géneros Pediobius (2.49 \%) y Chrysocharis (0.83\%) se obtuvieron en bajas cantidades y su presencia no fue constante en todos los meses de muestreo (Fig. 2).

Especímenes de Tetrastichinae no se detectaron en julio de 2014 y julio de 2015, pero los géneros con mayor presencia fueron Galeopsomyia (6.08\%), que se recolectó en nueve meses de muestreo, con mayor cantidad en enero; Aprostocetus y Baryscapus se obtuvieron con el mismo porcentaje (3.04 \% cada uno), pero en diferentes meses y Tamarixia (2.76\%) se encontró únicamente en tres meses, recolectándose más en abril de 2015 (Fig. 3).

\section{DISCUSIÓN}

La entomofauna de Eulophidae, a nivel de género en la huerta de naranja estudiada, fue diversa e importante; lo que probablemente indica múltiples y complejas interacciones. Este conjunto de géneros seguramente jugó un papel ecológico muy importante en los cítricos, ya que mantuvieron bajo control varias especies de artrópodos plaga. Los géneros Cirrospilus, Pnigalio, Sympiesis, $\mathrm{Ba}$ ryscapus, Aprostocetus, Horismenus, Pediobius y Chrysocharis se reportan como parasitoides del minador de la hoja de los cítricos Phyllocnistis citrella Stainton, 1856 (Lepidoptera: Gracillariidae) (Schauff et al., 1998; Gates et al., 2002). También se reporta a los géneros Elachertus, Miotropis, Euderus (Gates et al., 2002), Tetrastichus, Galeopsomyia y Elasmus (Schauff et al., 1998) como parasitoides del minador de la hoja de los cítricos. En México, Avedaño et al., (2005) reportaron a los géneros Cirrospilus, Elasmus, Quadrastichus y Horismenus como parasitoides de esta misma plaga. Por otro lado Tamarixia radiata Waterston, 1922 ha sido ampliamente utilizada en programas de control biológico contra el psílido asiático de los cítricos Diaphorina citri Kuwayama, 1907 (Gavarra et al., 1990), en México se ha realizado una campaña a gran escala para combatir al psílido por medio de la liberación masiva de $T$. radiata, donde se han producido y liberado más de 21.9 millones de parasitoides (SAGARPA, 2014).

Especies del género Pentastichus han sido asociadas a agallas en especies forestales y algunas del género Paragaleopsomyia se han encontrado en agallas de Cecidomyiidae; mientras que otras actúan como fitófagas formadoras de agallas (LaSalle, 1994). Las especies del

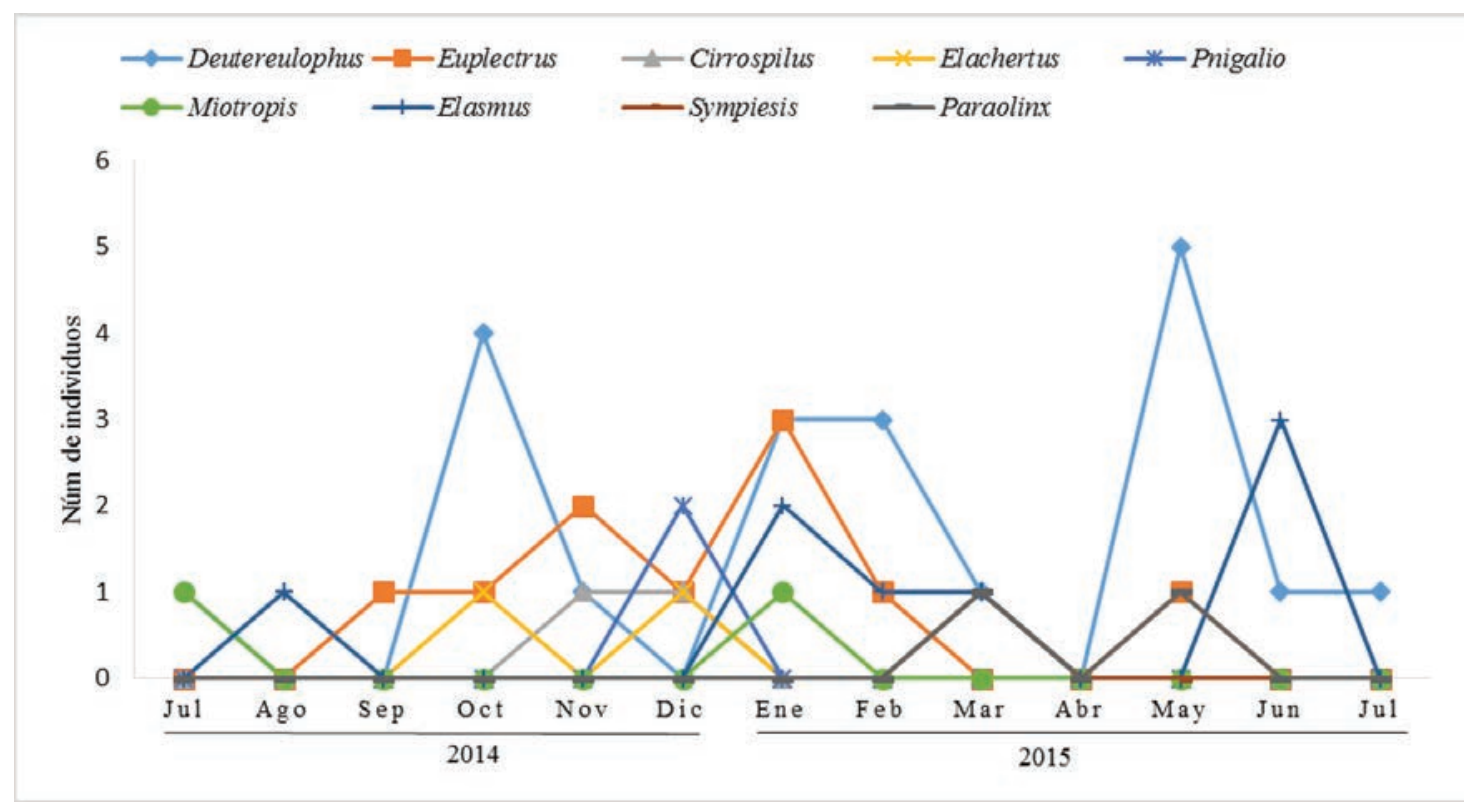

Figura 1. Distribución de la población en el tiempo de los géneros de Eulophinae 2014-2015. 


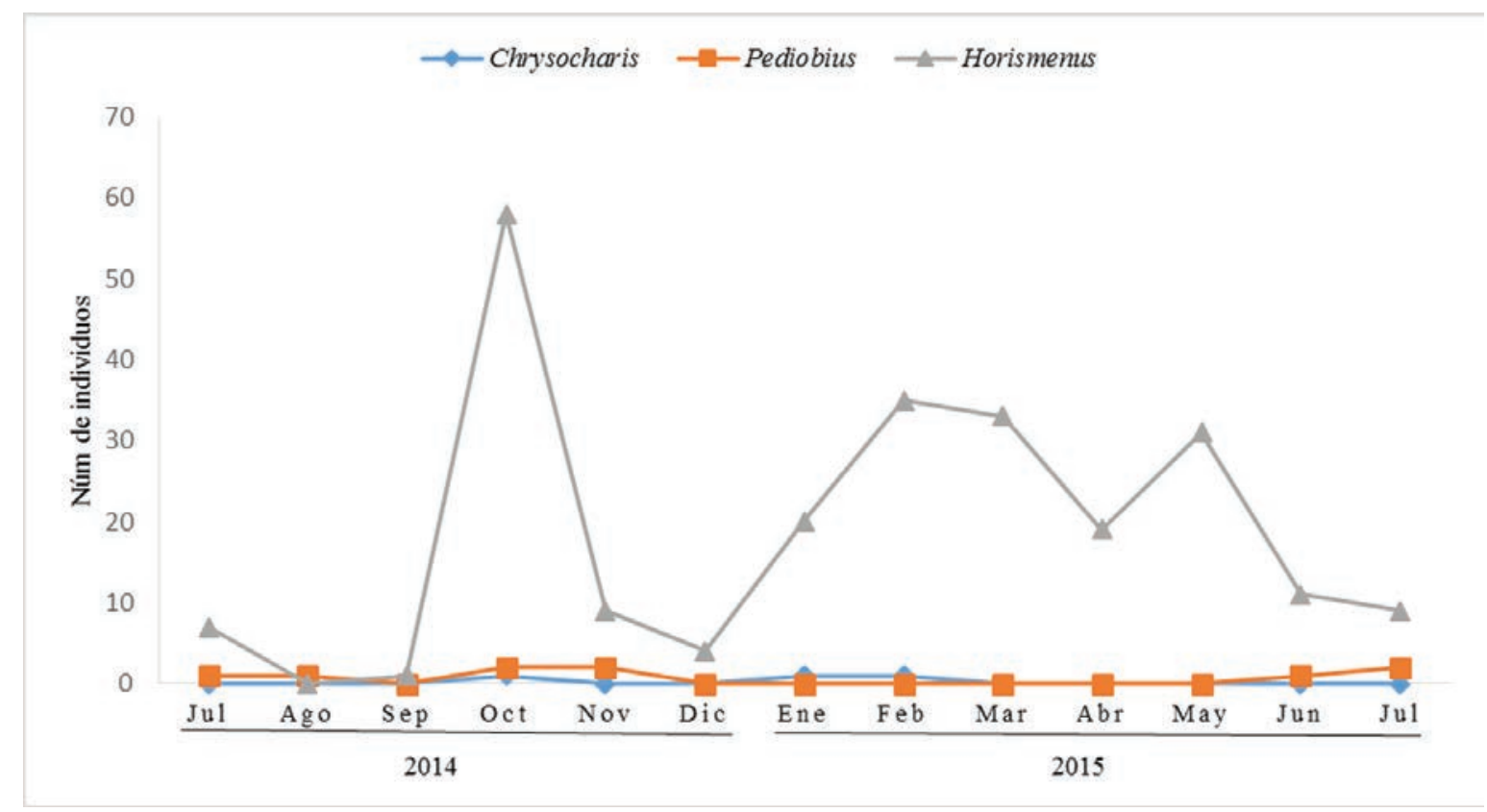

Figura 2. Distribución en el tiempo de la población de los géneros de Entedoninae 2014-2015.

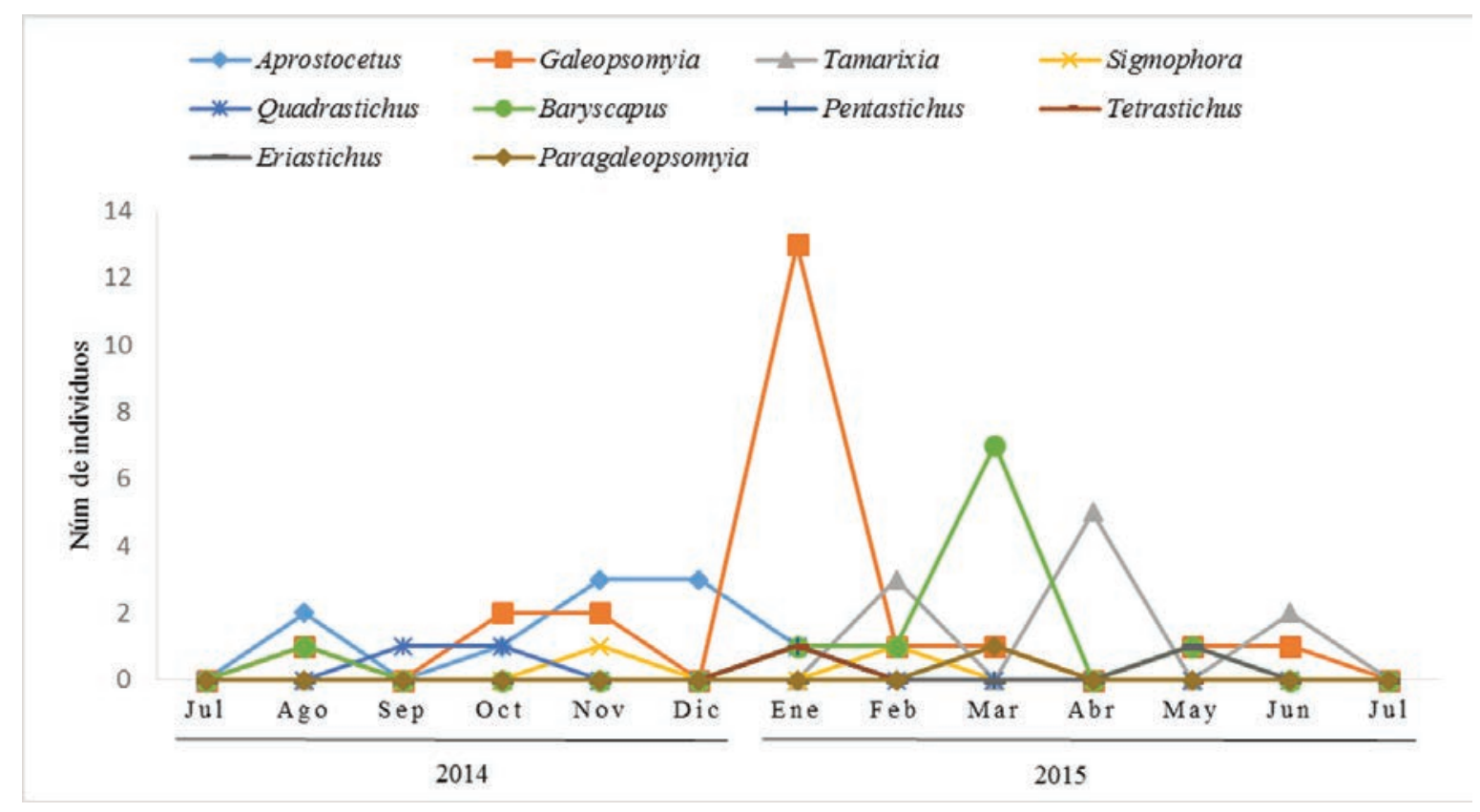

Figura 3. Distribución en el tiempo de los géneros de Tetrastichinae 2014-2015.

género Sigmophora pueden comportarse como ectoparasitoides, endoparasitoides o hiperparasitoides de dípteros, coleópteros e himenópteros (Yefremova \& Yegorenkova, 2009). Varias especies de Euplectrus se han reportado como parasitoides de lepidópteros que incluyen a las familias
Tortricidae, Noctuidae, Erebidae, entre otras (Hansson et al., 2015). Ghahari et al., (2016) consideran que es necesario conocer y conservar la fauna benéfica en los distintos ecosistemas, incluyendo los agrícolas, con la finalidad de incrementar la eficiencia de estos organismos. 


\section{LITERATURA CITADA}

Avedaño, G. F. J., Equihua M. A., Carrillo S, J. L. \& Bautista M. N. (2005). Identificación de parasitoides y nivel de parasitismo en Phyllocnistis citrella Stainton (Lepidoptera: Gracillariidae) en limón mexicano en Nueva Italia y Zicurán, Michoacán, México. Vedalia, 12, 129-138.

Burks, R. A. (2003). Key to the Nearctic genera of Eulophidae, subfamilies Entedoninae, Euderinae, and Eulophinae (Hymenoptera: Chalcidoidea). Available at: http://cache.ucr.edu/ heraty/Eulophidae/index.html (accessed on October 2016).

Burks, R. A., Heraty, J. M., Gebiola, M. \& Hansson, C. (2011). Combined molecular and morphological phylogeny of Eulophidae (Hymenoptera: Chalcidoidea), with focus on the subfamily Entedoninae. Cladistics, 27, 581-605.

Fronfría, M. A. (2003). Citricultura. 2a Edición. Ediciones MundiPrensa Libros. España, 422 pp.

Gates, M. W., Heraty, J. M., Schauff, M.L., Wagner, D. L., Whitfield, J. B. \& Wahl, D. B. 2002. Survey of the parasitic Hymenoptera on leafminers in California. Journal of Hymenoptera Research, 11, 213-270.

Gauthier, N., Lasalle, J., Quicke, D. L. \& Godfray, H. C. J. (2000). Phylogeny of Eulophidae (Hymenoptera: Chalcidoidea), with a reclassification of Eulophinae and the recognition that Elasmidae are derived eulophids. Systematic Entomology, 25, 521-539.

Gavarra, M. R., Mercado, B. G. \& Gonzales, C. I. (1990). Progress report: $D$. citri trapping, identification of parasite and possible field establishment of the imported parasite, Tamarixia radiata in the Philippines. In: Proceedings of the Fourth International Asia Pacific Conference on Citrus Rehabilitation. Chiang Mai, Thailand, 4-10.

Ghahari, H., Myartseva, S. N. \& Ruiz C. E. (2016). A faunistic study on some families of Chalcidoidea (Hymenoptera) from Golestan province and vicinity, Northern Iran. Folia Entomológica Mexicana, 2, 10-15.

Hansson, C. (2002). Eulophidae of Costa Rica (Hymenoptera: Chalcidoidea), 1. Memoirs of the American Entomological Institute, 67, 291.

Hansson, C. (2009). Eulophidae of Costa Rica (Hymenoptera: Chalcidoidea), 3 The Genus Horismenus. Memoirs of the American Entomological Institute, 82, 917.

Hansson, C., Smith, M. A., Janzen, D. H. \& Hallwachs, W. (2015).
Integrative taxonomy of New World Euplectrus Westwood (Hymenoptera, Eulophidae), with focus on 55 new species from Area de Conservación Guanacaste, northwestern Costa Rica. ZooKeys, 485, 1-236.

INEGI. (2009). Instituto Nacional de Estadística y Geografía. Prontuario de información geográfica municipal de los Estados Unidos Mexicanos. Tepalcingo, Morelos 2009. Available at: http://www3. inegi.org.mx/sistemas/mexicocifras/datos-geograficos/17/17019. pdf (accessed on August 2016).

LaSalle, J. (1994). North American genera of Tetrastichinae (Hymenoptera: Eulophidae). Journal of Natural History, 28, 109-236.

Noyes, J. S. (1982). Collecting and preserving chalcid wasps (Hymenoptera: Chalcidoidea). Journal of Natural Hystory, 16, 315-334.

Noyes, J. S. (2003). Universal Chalcidoidea Database. Available at: http://www.nhm.ac.uk/our-science/data/chalcidoids/introduction. html (accessed on August 2016).

SAGARPA. (2014). Secretaría de Agricultura, Ganadería, Desarrollo Rural, Pesca y Alimentación. Boletines. Disponible: http://sagarpa.gob.mx/saladeprensa/2012/Paginas/2014B695.aspx (consultado en Mayo 2016).

Schauff, M. E., LaSalle, J. \& Coote, L. D. (1997). Chapter 10 Eulophidae. Pp. 327-429. In: Gibson, G.A.P., Huber J.T., Woolley J.B (Eds). Annotated Keys to the Genera of Neartic Chalcidoidea (Hymenoptera). NRC Research Press, Otawa, Ontario, Canada.

Schauff, M. E., LaSalle, J. \& Wijesekara, G. A. (1998). The genera of chalcid parasitoids (Hymenoptera: Chalcidoidea) of citrus leafminer Phyllocnistis citrella Stainton (Lepidoptera: Gracillariidae). Journal of Natural History, 32, 1001-1056.

SIAP. (2016). Servicio de Información Agroalimentaria y Pesquera. Estadísticas. Disponible en: http://infosiap.siap.gob.mx:8080/ agricola_siap_gobmx/AvanceNacionalCultivo.do (consultado en September 2016).

Sosa-Armenta, J., López-Martínez, V., Jiménez-García, D. \& Alia, Y. (2012). Diaphorina citri Kuwayama (Hemiptera: Psyllidae) in Commercial Citrus Orchards in Morelos State, Mexico. Southwestern Entomologist, 37, 517-520.

Yefremova, Z. A. (2007). The subfamilies Eulophinae, Euderinae and Entedoninae (Hymenoptera: Eulophidae) in Yemen. Fauna of Arabia, 23, 335-368.

Yefremova, Z. A. \& Yegorenkova, E. N. (2009). A review of the subfamily Tetrastichinae (Hymenoptera: Eulophidae) in Yemen, with descriptions of new species. Fauna of Arabia, 24, 169-210. 